\title{
Close to the edge of Fundamental Measure Theory: density functional for hard sphere mixtures
}

\author{
José A Cuesta†, Yuri Martínez-Ratón† and \\ Pedro Tarazonat \\ † Grupo Interdisciplinar de Sistemas Complicados (GISC), Departamento de \\ Matemáticas, Universidad Carlos III de Madrid, Avda. de la Universidad, 30, \\ E-28911 Leganés, Madrid, Spain \\ ¥ Departamento de Física Teórica de la Materia Condensada, Universidad \\ Autónoma de Madrid, E-28049 Madrid, Spain.
}

\begin{abstract}
We analyze the structure of the Fundamental Measure Theory for the free energy density functional of hard sphere mixtures. A comparative study of the different versions of the theory, and other density functional approaches, is done in terms of their generic form for the three-points direct correlation function, which shows clearly the main advantages and problems of the different approximations. A recently developed version for the monocomponent case is extended to mixtures of hard spheres with different radii, and a new prescription is presented to obtain the exact dimensional crossover of those mixtures in the one-dimensional (1D) limit. Numerical results for planar wall-fluid interfaces and for the 1D fluid are presented.

PACS numbers: 05.20.Jj, 61.20.Gy, 61.20.Ne
\end{abstract}

\section{Introduction}

In the recent developments of density functional (DF) approximations for the free energy of hard molecules the Fundamental Measure Theory (FMT), pioneered by Rosenfeld [1] and explored in different directions by other authors [2, 3, 4, 5], stands at a prominent position both because of its peculiar functional structure and because of the combination of success and pitfalls obtained with its different versions. In this paper we analyze the generic mathematical structure of the different density functional approximations and discuss its implication for the generalization of a recent version of FMT to mixtures of hard spheres with different radii.

The theories developed to approximate the interaction part of the free energy density functional $\Phi[\rho] \equiv \beta\left(F[\rho]-F_{\text {id }}[\rho]\right)$, with $\beta=\left(k_{B} T\right)^{-1}$, beyond the simplest local density and square gradient approximations, are all based on a similar scheme: the non-local dependence on the density distribution $\rho(\mathbf{r})$ appears through 'averaged' or 'weighted' densities defined as convolutions

$$
\bar{\rho}_{k}(\mathbf{r})=\int \mathrm{d} \mathbf{r}^{\prime} \rho\left(\mathbf{r}^{\prime}\right) w_{k}\left(\mathbf{r}-\mathbf{r}^{\prime}\right),
$$

with several $(k=0,1, .$.$) weight functions, w_{k}\left(\mathbf{r}-\mathbf{r}^{\prime}\right)$, which depends only on the relative position of two points. The density functional $\Phi[\rho]$ is then written as the volume integral of a function of $\rho(\mathbf{r})$ and/or the weighted densities $\bar{\rho}_{k}(\mathbf{r})$. Such 
structure of the usual approximations for $\Phi[\rho]$ contrasts with the generic exact density expansion of the free energy,

$$
\begin{aligned}
\Phi[\rho]= & \frac{1}{2} \int \mathrm{d} \mathbf{r}_{1} \rho\left(\mathbf{r}_{1}\right) \int \mathrm{d} \mathbf{r}_{2} \rho\left(\mathbf{r}_{2}\right) f\left(\mathbf{r}_{12}\right) \\
& +\frac{1}{6} \int \mathrm{d} \mathbf{r}_{1} \rho\left(\mathbf{r}_{1}\right) \int \mathrm{d} \mathbf{r}_{2} \rho\left(\mathbf{r}_{2}\right) \int \mathrm{d} \mathbf{r}_{3} \rho\left(\mathbf{r}_{3}\right) f\left(\mathbf{r}_{12}\right) f\left(\mathbf{r}_{23}\right) f\left(\mathbf{r}_{31}\right)+\mathrm{O}\left(\rho^{4}\right),
\end{aligned}
$$

in terms of the Mayer function $f(\mathbf{r})=1-\mathrm{e}^{-\beta \phi(\mathbf{r})}$ for any pair potential energy $\phi(\mathbf{r})$ (we have denoted $\mathbf{r}_{i j} \equiv \mathbf{r}_{i}-\mathbf{r}_{j}$ ). The first term in (1.2) has the simplest structure of the density functional approximations, with a basic weight function $w_{0}(\mathbf{r})$ proportional to $f(\mathbf{r})$, used to convolute $\rho(\mathbf{r})$ at two different points. However, the second and followings terms in (1.2) cannot be reproduced within the generic forms used in the DF approximations. In the diagrammatic expansion used in the theory of liquids [6], the free energy is given by the irreducible diagrams (with Mayer function links), which cannot be evaluated as a single volume integral of simpler factors, while the generic form of the DF approximations includes only reducible diagrams with $w_{k}(\mathbf{r})$ links.

The second and third functional derivatives of (1.2) give the exact series expansions for the pair and triplet direct correlations functions respectively, which have the corresponding density expansions,

$$
-c^{(2)}\left(\mathbf{r}_{1}, \mathbf{r}_{2}\right)=\frac{\delta^{2} \Phi[\rho]}{\delta \rho\left(\mathbf{r}_{1}\right) \delta \rho\left(\mathbf{r}_{2}\right)}=f\left(\mathbf{r}_{12}\right)+f\left(\mathbf{r}_{12}\right) \int \mathrm{d} \mathbf{r}_{3} \rho\left(\mathbf{r}_{3}\right) f\left(\mathbf{r}_{23}\right) f\left(\mathbf{r}_{31}\right)+\mathrm{O}\left(\rho^{2}\right),(1
$$

and

$$
-c^{(3)}\left(\mathbf{r}_{1}, \mathbf{r}_{2}, \mathbf{r}_{3}\right)=\frac{\delta^{3} \Phi[\rho]}{\delta \rho\left(\mathbf{r}_{1}\right) \delta \rho\left(\mathbf{r}_{2}\right) \delta \rho\left(\mathbf{r}_{2}\right)}=f\left(\mathbf{r}_{12}\right) f\left(\mathbf{r}_{23}\right) f\left(\mathbf{r}_{31}\right)+\mathrm{O}(\rho),
$$

again made of irreducible diagrams, with two and three open (not integrated) points respectively. The first term in (1.3) is reproduced by most DF approximations, but the first term in (1.4), and hence the second term in (1.3) for arbitrary density distributions, cannot be reproduced within those DF forms. This contradiction between the exact forms and the usual choices for the mathematical structure of the DF approximations is forced by the computational cost of evaluating irreducible terms. The inclusion of kernel functions with more than two centers in the convolutions of $\rho(\mathbf{r})$ would make it difficult to use the DF approximation for practical purposes.

The weighted density approximation (WDA) [7, 8] gives a good example of the compromise between accuracy and practical computability in DF approximations. Although the advanced versions of this DF approximation use a density dependent weight $w(r, \bar{\rho})$, which may appear to be beyond the scope of (1.1), for the present discussion it is fully equivalent to take the zeroth and first order contributions in the density expansion $w(r, \rho)=w_{0}(r)+w_{1}(r) \rho+\cdots$. Then, the approximation for $\Phi[\rho]$ up to third order in $\rho(\mathbf{r})$ is

$$
\begin{aligned}
\Phi_{\mathrm{WDA}} & {[\rho]=\frac{1}{2} \int \mathrm{d} \mathbf{r}_{1} \rho\left(\mathbf{r}_{1}\right) \int \mathrm{d} \mathbf{r}_{2} \rho\left(\mathbf{r}_{2}\right) f\left(\mathbf{r}_{12}\right) } \\
+ & \frac{1}{6} \int \mathrm{d} \mathbf{r}_{1} \rho\left(\mathbf{r}_{1}\right) \int \mathrm{d} \mathbf{r}_{2} \rho\left(\mathbf{r}_{2}\right) \int \mathrm{d} \mathbf{r}_{3} \rho\left(\mathbf{r}_{3}\right) f\left(\mathbf{r}_{12}\right)\left[f\left(\mathbf{r}_{23}\right)+w_{1}\left(\mathbf{r}_{23}\right)\right]+\mathrm{O}\left(\rho^{4}\right),
\end{aligned}
$$

where the first term reproduces directly the first term in (1.2), while the second term tries to mimic the irreducible kernels as reducible combinations of Mayer function links, $f(\mathbf{r})$, with a new link function, $w_{1}(\mathbf{r})$, defined to recover the direct correlation function of a bulk fluid, i.e. to recover the exact expansion (1.3) up to first order in the 
uniform density $\rho(\mathbf{r})=\rho_{0}$. This requirement leads to a function $w_{1}(\mathbf{r})$ which exceeds the range of the Mayer function with an oscillatory tail structure.

Thus, the WDA for the hard sphere (HS) fluid may be implemented [8] to recover the direct correlation of the bulk fluid, $c\left(r_{12}, \rho_{0}\right)=\left.c^{(2)}\left(\mathbf{r}_{1}, \mathbf{r}_{2}\right)\right|_{\rho(\mathbf{r})=\rho_{0}}$, given by the Percus-Yevick approximation [9, 6], with the range of a HS diameter, $\sigma$; however the function $c^{(2)}\left(\mathbf{r}_{1}, \mathbf{r}_{2}\right)$, evaluated for a non-uniform density distribution, would exceed that range because of the convolution of the Mayer function with the oscillatory function $w_{1}(\mathbf{r})$. Similarly, the zeroth order term of the triplet direct correlation $c^{(3)}\left(\mathbf{r}_{1}, \mathbf{r}_{2}, \mathbf{r}_{3}\right)$ would exceed the range $r_{i j}=\left|\mathbf{r}_{i}-\mathbf{r}_{j}\right| \leq \sigma$ which the exact form (1.4) has for any combination of its variables. This being so, the WDA and similar approximations give a blurred representation of the exact non-local dependence of $\Phi[\rho]$ on $\rho(\mathbf{r})$, where the sharp step-like dependence on the HS Mayer function in (1.2)-(1.4) is replaced by an easier to compute but smoother functional. The extension of this type of DF to mixtures of HS with different radii $R_{i}(i=1, \ldots, m)$ is difficult, because they require $m(m+1) / 2$ weight functions to reproduce the Mayer functions $f_{i j}(r)$ for any pair of molecules, and the 'extended' weights required to mimic the irreducible diagrams for any triplet $(i, j, k=1, \ldots, m)$ proliferate into an embarrassing large number of possible combinations for the functional $\Phi[\rho]$.

The FMT also approximates $\Phi[\rho]$ for hard sphere systems in terms of convolutions (1.1) of $\rho(\mathbf{r})$, but with weight functions having the range of the molecular radius, $R$, rather than the range of the Mayer function, $\sigma=2 R$. The first advantage of such a choice is a natural extension to HS mixtures, since the number of different weight functions is going to be proportional to the number of species, and (at least in the original version) they only appear in a fixed number of linear combinations. The first one is the local packing fraction, which for a mixture of $(i=1, \ldots, m)$ species of hard spheres with radii $R_{i}$ and density distributions $\rho_{i}(\mathbf{r})$, is defined as

$$
\eta(\mathbf{r})=\sum_{i=1}^{m} \int \mathrm{d} \mathbf{r}^{\prime} \rho_{i}\left(\mathbf{r}^{\prime}\right) \Theta\left(R_{i}-\left|\mathbf{r}-\mathbf{r}^{\prime}\right|\right)
$$

with the Heaviside step function $\Theta(x)$. This definition of $\eta(\mathbf{r})$ is the direct extension to inhomogeneous systems of the usual packing fraction $\eta=(4 \pi / 3) \sum_{i} R_{i}^{3} \rho_{i}$, which plays a most relevant role in the best approximations for the equation of state of bulk fluid HS mixtures. Also, $\eta(\mathbf{r})$ is a key ingredient in the exact density functional for hard rods [10] in one dimension (1D), and it has the appealing interpretation of being the probability that point $\mathbf{r}$ happens to be inside a sphere. Despite these facts, the local packing fraction cannot appear in any DF based on the Mayer function and its convolutions, like the WDA in (1.5), because all the weight functions have at least the range of two added molecular radii. The problem to include $\eta(\mathbf{r})$ in the functional dependence of $\Phi[\rho]$ is to recover the Mayer function in the lowest order terms in (1.2) and (1.3), from kernels with half the range. In the exact density functional for hard rods in $1 \mathrm{D}$, the function $f_{i j}(x)=\Theta\left(R_{i}+R_{j}-|x|\right)$ is recovered as a convolution of the 'molecular volume' $\Theta\left(R_{i}-|x|\right)$ with delta functions,

$f_{i j}(x)=\frac{1}{2} \int \mathrm{d} x^{\prime}\left[\Theta\left(R_{i}-\left|x-x^{\prime}\right|\right) \delta\left(R_{j}-\left|x^{\prime}\right|\right)+\Theta\left(R_{j}-\left|x-x^{\prime}\right|\right) \delta\left(R_{i}-\left|x^{\prime}\right|\right)\right]$,

which points to the use of normalized spherical delta function shells as 'molecular surface' weight functions to complement $\eta(\mathbf{r})$.

In 3D the minimal set of weight functions with range $R_{i}$ and $R_{j}$ to recover the Mayer function spherical step at $|\mathbf{r}|=R_{i}+R_{j}$, as in (1.7) for the 1D case, requires a 
scalar and a vector weight functions to define two sets of weighted densities:

$$
n_{i}(\mathbf{r})=\frac{1}{4 \pi R_{i}^{2}} \int \mathrm{d} \mathbf{r}^{\prime} \delta\left(\left|\mathbf{r}^{\prime}\right|-R_{i}\right) \rho_{i}\left(\mathbf{r}+\mathbf{r}^{\prime}\right)
$$

and

$$
\mathbf{v}_{i}(\mathbf{r})=\frac{1}{4 \pi R_{i}^{2}} \int \mathrm{d} \mathbf{r}^{\prime} \delta\left(\left|\mathbf{r}^{\prime}\right|-R_{i}\right) \rho_{i}\left(\mathbf{r}+\mathbf{r}^{\prime}\right) \frac{\mathbf{r}^{\prime}}{R_{i}},
$$

for each molecular species $(i=1, \ldots, m)$ present in the system with density $\rho_{i}(\mathbf{r})$. The structure of the density functional $\Phi[\rho]$ at quadratic order in $\eta(\mathbf{r}), n_{i}(\mathbf{r})$ and $\mathbf{v}_{i}(\mathbf{r})$ is uniquely determined to recover the leading terms in $(1.2)$ and $(1.3)$,

$$
\begin{gathered}
\Phi[\rho]=\int \mathrm{d} \mathbf{r}\left(\eta(\mathbf{r}) \sum_{i=1}^{m} n_{i}(\mathbf{r})+2 \pi \sum_{i, j=1}^{m} R_{i} R_{j}\left(R_{i}+R_{j}\right)\left[n_{i}(\mathbf{r}) n_{j}(\mathbf{r})-\mathbf{v}_{i}(\mathbf{r}) \cdot \mathbf{v}_{j}(\mathbf{r})\right]\right) \\
+\mathrm{O}\left(\rho^{3}\right),
\end{gathered}
$$

The original proposal for the FMT [1] is consistent with (1.10) and, with the guideline of the scaled particle theory, recovers the full Percus-Yevick (PY) approximation for the direct correlation function of a bulk HS fluid. According with an general rule emanating from the FMT structure, the free energy density functional has as many additive terms as the space dimension,

$$
\Phi[\rho]=\sum_{i=1}^{D} \Phi_{i}^{(D)}[\rho] .
$$

For 3D hard spheres the three terms proposed by Rosenfeld were

$$
\begin{aligned}
& \Phi_{1}[\rho]=-\int \mathrm{d} \mathbf{r} \log [1-\eta(\mathbf{r})] \sum_{i=1}^{m} n_{i}(\mathbf{r}), \\
& \Phi_{2}[\rho]=2 \pi \sum_{i, j=1}^{m} R_{i} R_{j}\left(R_{i}+R_{j}\right) \int \mathrm{d} \mathbf{r} \frac{n_{i}(\mathbf{r}) n_{j}(\mathbf{r})-\mathbf{v}_{i}(\mathbf{r}) \cdot \mathbf{v}_{j}(\mathbf{r})}{1-\eta(\mathbf{r})}, \\
& \Phi_{3, \mathrm{o}}[\rho]=8 \pi^{2} \sum_{i, j, k=1}^{m} R_{i}^{2} R_{j}^{2} R_{k}^{2} \int \mathrm{d} \mathbf{r}_{i} n_{i}(\mathbf{r}) \frac{\frac{1}{3} n_{j}(\mathbf{r}) n_{k}(\mathbf{r})-\mathbf{v}_{j}(\mathbf{r}) \cdot \mathbf{v}_{k}(\mathbf{r})}{[1-\eta(\mathbf{r})]^{2}} .
\end{aligned}
$$

All the complexity arising from the mixture of $m$ different HS species is reduced to the evaluation of $\eta(\mathbf{r})$ in (1.6) and three moments $X^{(s)}(\mathbf{r})=\sum_{i} R_{i}^{s} X_{i}(\mathbf{r})$, with $s=0,1,2$, of the averaged densities $X_{i}=n_{i}, \mathbf{v}_{i}$. Thus, the theory may be applied even to polydisperse systems with a continuous distribution of the molecular radius [11].

For one dimensional (1D) hard rods the exact free energy DF, as found by Percus [10], is recovered from just the first term in (1.12), with the obvious translation of $\eta(\mathbf{r})$ and $n_{i}(\mathbf{r})$ to $1 \mathrm{D}$. For hard disks in two dimensions (2D), Rosenfeld [12] proposed two terms with structures similar to (1.12)-(1.13). The results obtained with this approximation for a monocomponent HS system were very good (better than those obtained with any previous DF approximation) for problems like determining the profiles of a HS fluid against a hard wall, thus showing that the non-local dependence obtained with the geometric measures (1.6), (1.8) and (1.9) is a better representation of the sharp dependence of the Mayer function in the irreducible kernels of (1.2). However, the study of the HS crystal as a density distribution made of narrow peaks at the positions of the crystal lattice, which had been successfully achieved with the WDA [7, 8] and other DF approximations [13], gave fully unphysical results 
with a negatively diverging free energy in the limit of infinitely narrow peaks. The problem was related to the overlap of three delta-function shells in (1.14), giving rise to integrable divergences. These singularities are harmless in the evaluation of the bulk fluid free energy and direct correlation function, but they notoriously show up when the free energy is evaluated for delta-function density distributions. In the case of a monocomponent HS system, the pathology of the original FMT was analyzed in terms of the dimensional crossover of the DF from 3D hard spheres to 1D hard rods, as well as the zero dimension (0D) limit, i.e. narrow cavities which cannot contain more than one molecule [14, 15]. A new version of $\Phi_{3}[\rho]$ was proposed to solve the problem [16], with the new ingredient of a 2-rank tensor-weighted density $\mathcal{T}_{i}$, with cartesian components

$$
T_{i}^{(\alpha, \beta)}(\mathbf{r})=\frac{1}{4 \pi R_{i}^{2}} \int \mathrm{d} \mathbf{r}^{\prime} \delta\left(\left|\mathbf{r}^{\prime}\right|-R_{i}\right) \rho_{i}\left(\mathbf{r}+\mathbf{r}^{\prime}\right) \frac{r_{\alpha}^{\prime} r_{\beta}^{\prime}}{R_{i}^{2}},
$$

$\alpha, \beta=x, y, z$ and where the index $i$ is included to provide the obvious generalization to HS mixtures. This tensor-weighted density allows to write $\Phi_{3}[\rho]$ in such a way that it vanishes for any $1 \mathrm{D}$ distribution of HS, i.e. for any $\rho(\mathbf{r})=\delta(x) \delta(y) \rho_{1}(z)$, which should be fully equivalent to a $1 \mathrm{D}$ system of hard rods with density distribution $\rho_{1}(z)$. The combined forms of $\Phi_{1}[\rho]$ and $\Phi_{2}[\rho]$ give the exact DF form in this limit, but the original term $\Phi_{3}[\rho]$ in (1.14) spoils the agreement. The new version of the FMT for the monocomponent HS system reproduces the exact 1D limit and gives an excellent description of the HS crystal, solving many of the qualitative problems of other DF approximations [16].

The extension of this new version of FMT to HS mixtures amounts to taking

$$
\Phi_{3}[\rho]=12 \pi^{2} \sum_{i, j, k=1}^{m} R_{i}^{2} R_{j}^{2} R_{k}^{2} \int \mathrm{d} \mathbf{r} \frac{\varphi_{i j k}(\mathbf{r})}{[1-\eta(\mathbf{r})]^{2}},
$$

with

$$
\varphi_{i j k}(\mathbf{r})=\mathbf{v}_{i} \cdot \mathcal{T}_{j} \cdot \mathbf{v}_{k}-n_{j} \mathbf{v}_{i} \cdot \mathbf{v}_{k}-\operatorname{Tr}\left[\mathcal{T}_{i} \mathcal{T}_{j} \mathcal{T}_{k}\right]+n_{j} \operatorname{Tr}\left[\mathcal{I}_{i} \mathcal{T}_{k}\right],
$$

in terms of the tensorial contractions and trace of $\mathbf{v}_{i}(\mathbf{r})$ and $\mathcal{T}_{i}(\mathbf{r})$, for each component.

However, such a direct extension to mixtures fails to reproduce the exact 1D limit. The reason for this failure, the modification in the DF structure required to achieve that limit and the effect of such modifications in typical problems are to be analyzed here. In the next section, the origin and effects of the spurious divergences in the FMT density functional are analyzed in terms of the triplet direct correlation function and a new FMT density functional for HS mixtures is proposed to recover the exact 1D limit. This new version, however, patches out this effect at the expense of creating new weaker but potentially harmful singularities which preclude using this functional for a free minimization. In section 3 we compare the results of this new version with those obtained with (1.16) and (1.17), and discuss the pros and cons of using each. We conclude in section 4 that the functional (1.16), (1.17), despite its defects, provides the best balance between accuracy and computational simplicity, and that it seems impossible to go any further without modifying the basic structure of FMT.

\section{FMT for HS mixtures}

The triplet direct correlation functions obtained for HS in three dimensions with any FMT density functional has a peculiar structure, very different from that obtained 


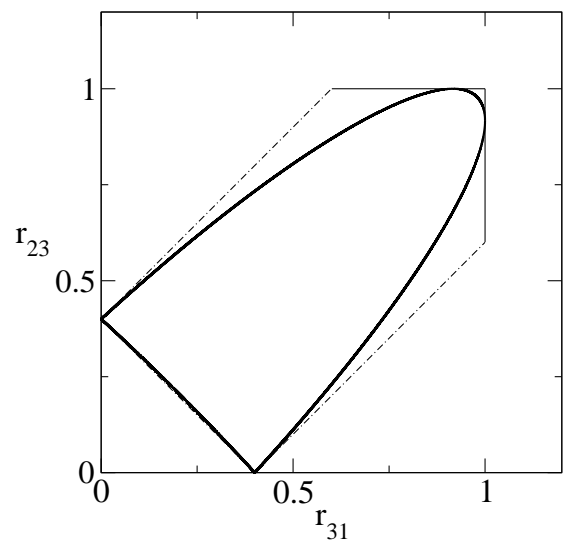

(a)

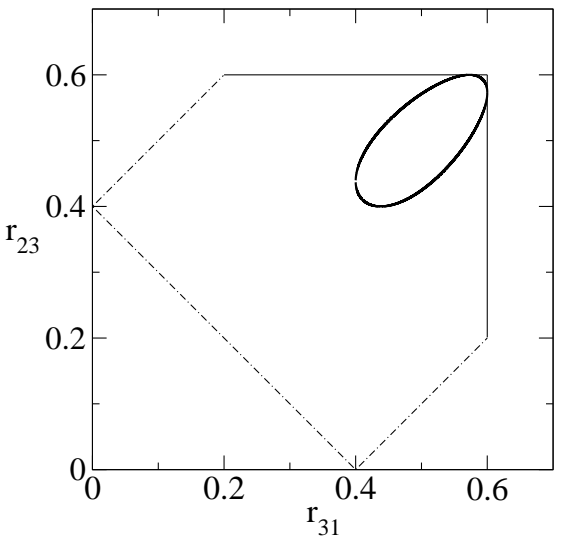

(b)

Figure 1. Boundaries in the plane $\left(r_{23}, r_{31}\right)$, for the given value of $r_{12}=0.4$. The dash-dotted lines give the triangle inequalities $\left|r_{23}-r_{31}\right| \leq r_{12} \leq r_{23}+r_{31}$, for the possible values of these variables. The solid thick curves give the regions of common overlap between three spheres placed at $\mathbf{r}_{1}, \mathbf{r}_{2}$ and $\mathbf{r}_{3}$ while the solid thin lines give the boundaries of the simultaneous pairwise overlap. The HS radii are of equal size $\left(R_{i}=R_{j}=R_{k}=0.5\right)$ (a) and of very different size $\left(R_{i}=R_{j}=0.5\right.$ and $R_{k}=0.1$ ) (b).

with the WDA or similar approximations. The most relevant form appears in the contribution at zero order in a density expansion, from the functional derivative of $\Phi_{3}[\rho]$ in (1.11), which has the generic form

$$
\begin{aligned}
\frac{\delta^{3} \Phi_{3}[\rho]}{\delta \rho_{i}\left(\mathbf{r}_{1}\right) \delta \rho_{j}\left(\mathbf{r}_{2}\right) \delta \rho_{k}\left(\mathbf{r}_{3}\right)}= & \int \mathrm{d} \mathbf{r} \delta\left(R_{i}-\left|\mathbf{r}_{1}^{\prime}\right|\right) \delta\left(R_{j}-\left|\mathbf{r}_{2}^{\prime}\right|\right) \delta\left(R_{k}-\left|\mathbf{r}_{3}^{\prime}\right|\right) K_{i j k}\left(\mathbf{r}_{1}^{\prime}, \mathbf{r}_{2}^{\prime}, \mathbf{r}_{3}^{\prime}\right) \\
& +\mathrm{O}(\rho),
\end{aligned}
$$

where $i, j, k=1, \ldots, m$. The radii of the HS placed at $\mathbf{r}_{1}, \mathbf{r}_{2}$ and $\mathbf{r}_{3}$ are $R_{i}, R_{j}$ and $R_{k}$ respectively, and for each possible choice of the three indices, $i, j, k$, there is a generic function $K_{i j k}$ of the relative vectors $\mathbf{r}_{l}^{\prime} \equiv \mathbf{r}_{l}-\mathbf{r}, l=1,2,3$, whose moduli are restricted to be equal to the respective HS radii by the three deltafunction shells. Higher order terms in the density expansion of $c_{i j k}^{(3)}$, as well as the contributions to the triplet direct correlation function from the terms $\Phi_{1}[\rho]$ and $\Phi_{2}[\rho]$ in (1.12) and (1.13), have one or more delta-function shells substituted by spherical step functions (i.e. $\Theta\left(R_{i}-\left|\mathbf{r}_{1}^{\prime}\right|\right)$, etc.) coming from the functional derivative of $\eta(\mathbf{r})$. These contributions are always regular smooth functions, which cannot produce spurious divergences but cannot reproduce either the exact step-like dependence of $c_{i j k}\left(\mathbf{r}_{1}, \mathbf{r}_{2}, \mathbf{r}_{3}\right)=-f_{i j}\left(r_{12}\right) f_{j k}\left(r_{23}\right) f_{k i}\left(r_{31}\right)+\mathrm{O}(\rho)$, in terms of the Mayer functions, $f_{i j}(r)=\Theta\left(R_{i}+R_{j}-r\right)$, and the relative distances $r_{12}=\left|\mathbf{r}_{1}-\mathbf{r}_{2}\right|$.

It is only from the overlap of the three delta-function shells in (2.1) that the $c_{i j k}^{(3)}$ term in a FMT may be able to reproduce such a sharp step dependence. But the same overlap is also the origin of the spurious divergences which may invalidate the use of the FMT for density distributions with strong inhomogeneities. The crucial point to balance these two aspects is the choice of the kernel function $K_{i j k}\left(\mathbf{r}_{1}^{\prime}, \mathbf{r}_{2}^{\prime}, \mathbf{r}_{3}^{\prime}\right)$, which is precisely the difference between different versions of the FMT. The common aspect of all versions is that the range of the triplet correlation function, $c_{i j k}^{(3)}\left(\mathbf{r}_{1}, \mathbf{r}_{2}, \mathbf{r}_{3}\right)$, is 
restricted to points $\mathbf{r}_{1}, \mathbf{r}_{2}$ and $\mathbf{r}_{3}$ such that the three spheres of radii $R_{i}, R_{j}$ and $R_{k}$, respectively centered at those points, have a common overlap. This geometrical boundary is always inside the exact range imposed by the Mayer functions product (which extends as far as pairwise overlaps between the three spheres exist) but, particularly in the case of mixtures of HS with very different sizes, it is much more restrictive than the exact one. In figure 1 we represent the boundaries in the plane $\left(r_{23}, r_{31}\right)$ for a given value of $r_{12}$, both for HS of equal size (a) and of very different size (b). The total hyper-volume obtained by integration over $\mathbf{r}_{2}$ and $\mathbf{r}_{3}$ in the region with non-zero values for (2.1) may be obtained as a function of the HS radii as $\Gamma_{\mathrm{FMT}}^{(3)}=4 \pi^{4} R_{i}^{2} R_{j}^{2} R_{k}^{2}$, while the equivalent integral for the exact product of Mayer functions gives

$$
\begin{aligned}
\Gamma_{\text {exact }}^{(3)}=\frac{16 \pi^{2}}{9}[ & R_{i}^{3} R_{j}^{3}+R_{j}^{3} R_{k}^{3}+R_{k}^{3} R_{i}^{3} \\
& \left.+3 R_{i}^{2} R_{j}^{2} R_{k}^{2}\left(3+\frac{R_{i}+R_{j}}{R_{k}}+\frac{R_{j}+R_{k}}{R_{i}}+\frac{R_{k}+R_{i}}{R_{j}}\right)\right] .
\end{aligned}
$$

The difference between $\Gamma_{\text {exact }}^{(3)}$ and $\Gamma_{\mathrm{FMT}}^{(3)}$ corresponds to the FMT 'lost-cases' described in the 0D approach to the FMT [15], i.e. those configurations of three delta-function peaks which cannot give contribution to $\Phi_{3}[\rho]$ in the FMT scheme, but which should give a contribution to the exact free energy excess. When the three HS radii are equal the FMT contribution covers about $74 \%$ of the exact hyper-volume, so that the role of the 'lost-cases' is relatively mild, and its effect on the overall accuracy of the DF approximation for usual applications could be reduced by an appropriate choice of the kernel function in (2.1), which could increase the contribution of the points inside the FMT boundary to overcome the lack of contribution of the missing configurations. However, the ratio between $\Gamma_{\mathrm{FMT}}^{(3)}$ and $\Gamma_{\text {exact }}^{(3)}$ goes to zero in a mixture of very asymmetric HS, whenever the size of one species goes to zero while keeping the size of the other two. This is shown in figure 2 and corresponds to the effect which was obvious for the particular case in figure 1 1 (b): the contribution of $\Phi_{3}[\rho]$ associated to spheres of very different size shrinks to a very small part of the exact contribution to the triplet direct correlation function. If the kernel function in (2.1) is fixed to reproduce a given equation of state for the bulk fluid mixture, the contribution of order $\rho_{i} \rho_{j} \rho_{k}$ coming from $\Phi_{3}[\rho]$ would require an enormous artificial enhancement of the weight of those configuration within the FMT boundary, in order to compensate the lack of contribution from the missing configurations. The use of such an approximation for inhomogeneous density distributions could lead to quite an erroneous evaluation of the excess free energy, with spurious sensitivity to the density distribution of the small spheres. This could be of particular relevance in the DF study of depletion forces in colloidal particles for strongly inhomogeneous density distributions.

Beside the problem of its reduced range, the mathematical form (2.1) leads to the most serious problem of the FMT: the presence of spurious divergences at the boundary of non-zero values, arising from the tangency of two or the three spherical shells. This is clear if the integral over $\mathbf{r}$ is transformed into integrals over the three moduli $r_{1}^{\prime}, r_{2}^{\prime}$ and $r_{3}^{\prime}$, which can be directly performed thanks to the three delta functions in (2.1). The boundary of non-zero values is therefore given by the existence of a triple overlap between the three spheres; since at that boundary the vectors $\mathbf{r}_{1}^{\prime}$, 


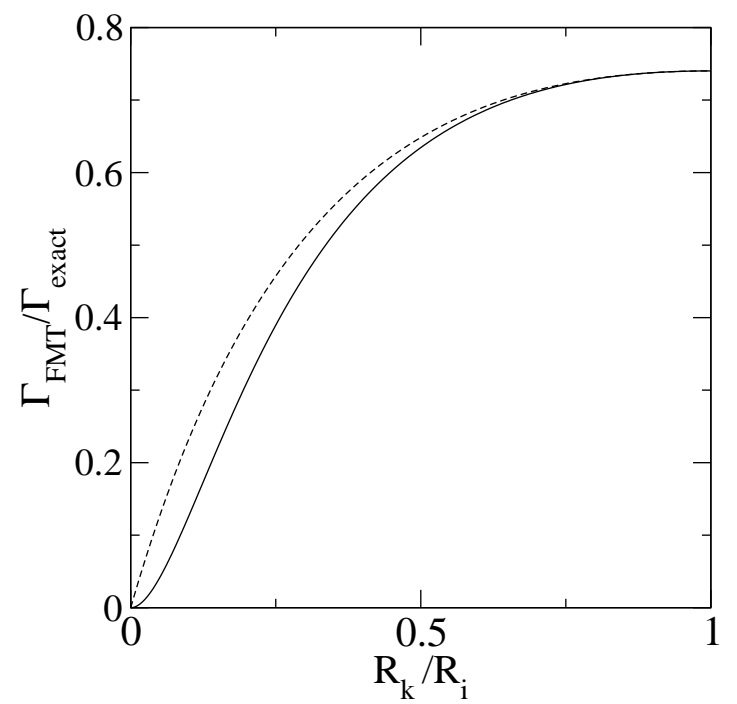

Figure 2. Ratio between $\Gamma_{\mathrm{FMT}}^{(3)}=4 \pi^{4} R_{i}^{2} R_{j}^{2} R_{k}^{2}$ and $\Gamma_{\text {exact }}^{(3)}$ [equation (2.2)] for $R_{j}=R_{i} \geq R_{k}$ (solid line) and for $R_{j}=R_{k} \leq R_{i}$ (dashed line).

$\mathbf{r}_{2}^{\prime}$ and $\mathbf{r}_{3}^{\prime}$ are geometrically fixed, integration leads to

$\frac{\delta^{3} \Phi_{3}[\rho]}{\delta \rho_{i}\left(\mathbf{r}_{1}\right) \delta \rho_{j}\left(\mathbf{r}_{2}\right) \delta \rho_{k}\left(\mathbf{r}_{3}\right)}=\frac{4 R_{i} R_{j} R_{k} K_{i j k}\left(\mathbf{r}_{1}^{\prime}, \mathbf{r}_{2}^{\prime}, \mathbf{r}_{3}^{\prime}\right)}{\left[Z\left(r_{12}, r_{23}, r_{31}, R_{i}, R_{j}, R_{k}\right)\right]^{1 / 2}}+\mathrm{O}(\rho)$.

The function $Z\left(r_{12}, r_{23}, r_{31}, R_{i}, R_{j}, R_{k}\right)$ is the polynomial

$$
\begin{aligned}
Z= & \left(R_{i}^{2} r_{23}^{2}+R_{j}^{2} R_{k}^{2}\right)\left(r_{12}^{2}-r_{23}^{2}+r_{31}^{2}\right)+\left(R_{j}^{2} r_{31}^{2}+R_{k}^{2} R_{i}^{2}\right)\left(r_{23}^{2}-r_{31}^{2}+r_{12}^{2}\right) \\
& +\left(R_{k}^{2} r_{12}^{2}+R_{i}^{2} R_{j}^{2}\right)\left(r_{31}^{2}-r_{12}^{2}+r_{23}^{2}\right)-R_{i}^{4} r_{23}^{2}-R_{j}^{4} r_{31}^{2}-R_{k}^{4} r_{12}^{2}-r_{12}^{2} r_{23}^{2} r_{31}^{2}
\end{aligned}
$$

arising from the Jacobian of the transformation. This polynomial vanishes over the whole boundary. The divergences of the terms in (2.1) and (2.3) may only be avoided if the kernel also vanishes over the boundary. The version of the FMT based on the exact 0D limit for narrow cavities of arbitrary shape 15], fulfills precisely such a condition with a function $K_{i j k}\left(\mathbf{r}_{1}^{\prime}, \mathbf{r}_{2}^{\prime}, \mathbf{r}_{3}^{\prime}\right)$ proportional to $\left[Z\left(r_{12}, r_{23}, r_{31}, R_{i}, R_{j}, R_{k}\right)\right]^{1 / 2}$. This removes the divergences in (2.3) while at the same time saves the step-like dependence between the inside and the outside of the FMT boundary. However, such a kernel function is not separable in powers of the cartesian components of the vectors $\mathbf{r}_{l}^{\prime}$, so that the free energy cannot be evaluated in terms of simple weighted densities, like (1.1), making it very costly to use it for practical applications.

The original FMT version (1.14) corresponds to building a kernel function on the basis of the bulk fluid direct correlation (from the integration of $c_{i j k}^{(3)}$ with respect to one of the positions, as in (1.3)) and to get an easy computation of $\Phi[\rho]$ in terms of the scalar and vector weight densities (1.8) and (1.9). In that case the kernel function has to be decomposable in terms which are linear on each cartesian component of the vector variables. Within these constraints the unique choice was (1.14), which is equivalent to taking in (2.1) and (2.3) the kernel

$$
K_{i j k}=\frac{1}{24 \pi}\left(1-\frac{\mathbf{r}_{1}^{\prime} \cdot \mathbf{r}_{2}^{\prime}}{R_{i} R_{j}}-\frac{\mathbf{r}_{2}^{\prime} \cdot \mathbf{r}_{3}^{\prime}}{R_{j} R_{k}}-\frac{\mathbf{r}_{3}^{\prime} \cdot \mathbf{r}_{1}^{\prime}}{R_{k} R_{i}}\right) .
$$


However, such a choice for the kernel function does not eliminate any of the divergences at the boundaries of the tangent spherical shells. The strongest effect appears when $R_{i}=R_{j}=R_{k}$ and $\mathbf{r}_{1}=\mathbf{r}_{2}=\mathbf{r}_{3}$, so that the three spherical shells overlap in their whole surface. In that case (2.5) takes the value $-(12 \pi)^{-1}$, and there is a diverging negative contribution to the free energy. The divergence is integrable and it contributes to give the exact $\rho^{3}$ term in free energy (1.2) for uniform bulk fluid, but in a density distribution with narrow peaks (like in a $0 \mathrm{D}$ cavity or in a crystal phase) the negative divergence goes to $\Phi_{3}[\rho]$ in (1.14) and the approximation becomes useless. Also, in the crossover from the $3 \mathrm{D}$ hard spheres to the $1 \mathrm{D}$ system, there is a spurious contribution of the divergence in $\Phi_{3}[\rho]$, as given in (1.14), while the exact 1D density functional would be recovered with a vanishing $\Phi_{3}[\rho]$ for such density distributions.

The FMT version (1.16) and (1.17) was developed to recover the exact 1D limit for monocomponent HS systems 16]. It corresponds to choosing the kernel

$K_{i j k}=\frac{1}{16 \pi}\left[\left(1-\frac{\mathbf{r}_{1}^{\prime} \cdot \mathbf{r}_{2}^{\prime}}{R_{i} R_{j}}\right)\left(1-\frac{\mathbf{r}_{2}^{\prime} \cdot \mathbf{r}_{3}^{\prime}}{R_{j} R_{k}}\right)\left(1-\frac{\mathbf{r}_{3}^{\prime} \cdot \mathbf{r}_{1}^{\prime}}{R_{k} R_{i}}\right)-\frac{\left[\mathbf{r}_{1}^{\prime} \cdot\left(\mathbf{r}_{2}^{\prime} \times \mathbf{r}_{3}^{\prime}\right)\right]^{2}}{\left(R_{i} R_{j} R_{k}\right)^{2}}\right]$.

The presence of a quadratic dependence in the cartesian components of the vector variables implies the need of the tensor weighted density, with rank two, defined in (1.15), together with the scalar and vector weighted densities (1.8) and (1.9) used in (1.14). The kernel has two terms with different geometrical structure: one goes with the triple vector product $\mathbf{r}_{1}^{\prime} \cdot\left(\mathbf{r}_{2}^{\prime} \times \mathbf{r}_{3}^{\prime}\right)$ which becomes zero whenever the three vectors $\mathbf{r}_{1}^{\prime}, \mathbf{r}_{2}^{\prime}$ and $\mathbf{r}_{3}^{\prime}$ become coplanar. This happens always at the boundaries of the triple overlap of the three spherical shells in (2.1) so that this contribution to the kernel alone would get rid of all the spurious divergences generated by the vanishing of the denominator in (2.3). Unfortunately, a kernel function based only on this term would not reproduce the correct behavior for $c^{(2)}(r, \rho)$ in a homogeneous fluid. The other contribution in (2.6) becomes zero whenever two of the three vectors $\mathbf{r}_{l}^{\prime}$ become equal, which suppresses the strongest divergences of $c^{(3)}\left(\mathbf{r}_{1}, \mathbf{r}_{2}, \mathbf{r}_{3}\right)$ produced when two delta functions shells, of equal radii, overlap their full surfaces. In the monocomponent case, this is enough to make the full contribution of $\Phi_{3}[\rho]$ to vanish in the $1 \mathrm{D}$ limit, because three spherical shells of equal radii and with the centers along a straight line, cannot have a triple overlap unless two of them share the same center.

Thus, in a monocomponent HS system the FMT version with (1.16) is free of the strongest divergences in $c^{(3)}\left(\mathbf{r}_{1}, \mathbf{r}_{2}, \mathbf{r}_{3}\right)$, produced when $\mathbf{r}_{l}=\mathbf{r}_{m}$ and when the three points $\mathbf{r}_{1}, \mathbf{r}_{2}, \mathbf{r}_{3}$ are along a straight line (so that the spherical shells have a common axis). However, there are still weak divergences at the boundary points between overlapping and non-overlapping shells. These divergences would appear in $\Phi[\rho]$ for very peculiar density distributions, with three delta function peaks, normalized to contain less than one molecule among the three, and separated by distances such that the surfaces of molecules centered at the three sites would intersect in a single point. Of course such a density distribution would be very unlikely to appear unless the external potential acting on the system has been tailored on purpose, and in that case the error made by this FMT version would be of the opposite sign to the error made by (1.14) in the 0D limit. Instead of a negative divergence of the free energy (from the negative sign of the kernel (2.5) in such a limit), there would be a positive divergence of the free energy because the kernel (2.6) is always equal or larger than zero for coplanar vectors. A positive divergence in $\Phi_{3}[\rho]$ for a very peculiar density distribution would produce little harm because the minimization process of the free energy in the density functional formalism would just avoid such distribution functions. The problem is 


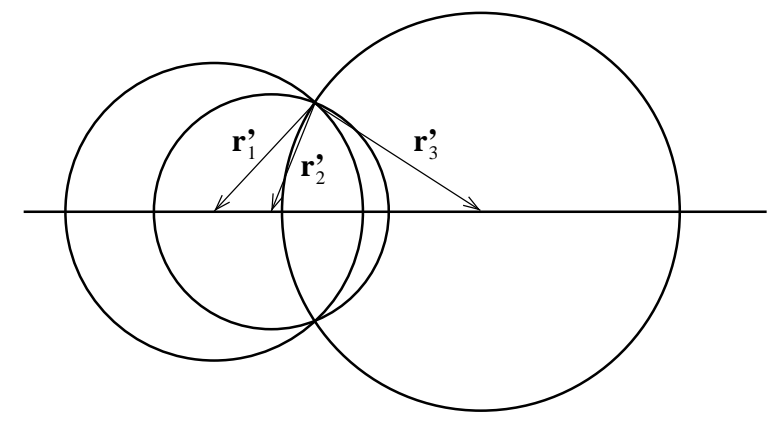

Figure 3. Three coaxial spheres of different radii overlapping along a circumference. This situation is impossible for spheres of the same radius, unless two of them overlap their whole surfaces.

much more innocuous than a negative divergence of the free energy, which would invalidate the formal use of $\Phi[\rho]$ to minimize with respect to any possible function $\rho(\mathbf{r})$.

In the case of HS mixtures with different radii, the kernel (2.6) is less efficient than in the monocomponent case in the regularization of the FMT. The exact 1D limit would still require that $\Phi_{3}[\rho]$ vanishes whenever all the points with non-zero density are along a straight line, but the kernel (2.6) does not vanish in the case of three coaxial spheres with a triple overlap along a circumference (as illustrated in figure 3). To recover the exact 1D density functional from a mixture of 3D hard spheres confined along a straight line requires a kernel $K_{i j k}\left(\mathbf{r}_{1}^{\prime}, \mathbf{r}_{2}^{\prime}, \mathbf{r}_{3}^{\prime}\right)$ which vanishes whenever the three vectors (with respective moduli $R_{i}, R_{j}$ and $R_{k}$ ) go from a common origin to three points along a straight line. The simplest geometrical construction with this requirement is

$$
\begin{aligned}
& K_{i j k}=\frac{\left(\mathbf{r}_{1}^{\prime} \times \mathbf{r}_{2}^{\prime}+\mathbf{r}_{2}^{\prime} \times \mathbf{r}_{3}^{\prime}+\mathbf{r}_{3}^{\prime} \times \mathbf{r}_{1}^{\prime}\right)}{16 \pi R_{i} R_{j} R_{k}} \cdot\left[\left(\frac{\mathbf{r}_{1}^{\prime} \cdot \mathbf{r}_{2}^{\prime}}{R_{i}+R_{j}}+\frac{\mathbf{r}_{1}^{\prime} \cdot \mathbf{r}_{3}^{\prime}}{R_{i}+R_{k}}\right) \frac{\left(\mathbf{r}_{3}^{\prime} \times \mathbf{r}_{2}^{\prime}\right)}{R_{k} R_{j}}+\right. \\
& \left.+\left(\frac{\mathbf{r}_{2}^{\prime} \cdot \mathbf{r}_{3}^{\prime}}{R_{j}+R_{k}}+\frac{\mathbf{r}_{2}^{\prime} \cdot \mathbf{r}_{1}^{\prime}}{R_{j}+R_{i}}\right) \frac{\left(\mathbf{r}_{1}^{\prime} \times \mathbf{r}_{3}^{\prime}\right)}{R_{i} R_{k}}+\left(\frac{\mathbf{r}_{3}^{\prime} \cdot \mathbf{r}_{1}^{\prime}}{R_{k}+R_{i}}+\frac{\mathbf{r}_{3}^{\prime} \cdot \mathbf{r}_{2}^{\prime}}{R_{k}+R_{j}}\right) \frac{\left(\mathbf{r}_{2}^{\prime} \times \mathbf{r}_{1}^{\prime}\right)}{R_{j} R_{i}}\right] .
\end{aligned}
$$

The way to obtain this kernel is to multiply the vector

$$
\mathbf{r}_{1}^{\prime} \times \mathbf{r}_{2}^{\prime}+\mathbf{r}_{2}^{\prime} \times \mathbf{r}_{3}^{\prime}+\mathbf{r}_{3}^{\prime} \times \mathbf{r}_{1}^{\prime}=\left(\mathbf{r}_{3}^{\prime}-\mathbf{r}_{2}^{\prime}\right) \times\left(\mathbf{r}_{1}^{\prime}-\mathbf{r}_{2}^{\prime}\right),
$$

which obviously vanish whenever the three vectors are coaxial, by some linear combination of the three cross products in such a way that the contribution to the triangle in the virial expansion of the direct correlation function is recovered. Such a thing cannot be accomplished if the coefficients are just functions of the radii, but it can if we allow for a dependence on the dot products of the vectors.

The existence of cubic dependence on the vectors in (2.7) forces to use a new 3-rank tensor measure, namely $\mathcal{M}_{i}$, whose cartesian components are given by

$$
M_{i}^{(\alpha, \beta, \gamma)}(\mathbf{r})=\frac{1}{4 \pi R_{i}^{2}} \int \mathrm{d} \mathbf{r}^{\prime} \delta\left(\left|\mathbf{r}^{\prime}\right|-R_{i}\right) \rho_{i}\left(\mathbf{r}+\mathbf{r}^{\prime}\right) \frac{r_{\alpha}^{\prime} r_{\beta}^{\prime} r_{\gamma}^{\prime}}{R_{i}^{3}} .
$$

In terms of this and the former measures, $\Phi_{3}[\rho]$ will have the same shape as in (1.16), 
but with $\varphi_{i j k}(\mathbf{r})$ in (1.17) replaced by $\varphi_{i j k}(\mathbf{r})+\Delta \varphi_{i j k}(\mathbf{r})$, where

$$
\Delta \varphi_{i j k}(\mathbf{r})=\frac{2 R_{j}^{2}\left(R_{i}-R_{k}\right)}{R_{i}\left(R_{i}+R_{j}\right)\left(R_{j}+R_{k}\right)}\left(\mathbf{v}_{i} \cdot \mathcal{M}_{j}: \mathcal{T}_{k}-\mathbf{v}_{i} \cdot \mathcal{T}_{k} \cdot \mathbf{v}_{j}\right),
$$

the symbol ' $:$ ' denoting the contraction of two indices. An important difference arises in this new version: the coefficients multiplying the measures are no longer a product of powers of the radii, but more general rational functions of them. This has the computational disadvantage of not allowing to express the functional in terms of a finite number of moments of the weighted densities, as in any previous FMT version. This is particularly important in applications to polydisperse system, where having moment dependent functionals dramatically simplifies the calculations [11.

A different and more formal drawback of the new kernel is the fact that it cancels the most singular terms in the former kernel (those that preclude it to recover the one-dimensional functional for aligned configurations of the HS) at the expense of changing the sign of the weak divergences for some non-aligned configurations, at the boundary between overlapping and non-overlapping shells. These divergences were always of positive sign for the kernel (2.6), but for some configurations they become negative with the kernel (2.7). This can be explicitly seen if we write the kernel (2.7) as

$$
K_{i j k}=\left[\left(\mathbf{r}_{3}^{\prime}-\mathbf{r}_{2}^{\prime}\right) \times\left(\mathbf{r}_{1}^{\prime}-\mathbf{r}_{2}^{\prime}\right)\right] \cdot V_{i j k}\left(\mathbf{r}_{1}^{\prime}, \mathbf{r}_{2}^{\prime}, \mathbf{r}_{3}^{\prime}\right),
$$

and now rotate $\mathbf{r}_{2}^{\prime}$ slightly away of coaxiality while maintaining the three vectors in the same plane and with common origin (see figure 3). If the displacement is given by the vector $\boldsymbol{\epsilon}\left(|\boldsymbol{\epsilon}| \ll R_{j}\right)$ then $R_{j}=\left|\mathbf{r}_{2}^{\prime}+\boldsymbol{\epsilon}\right|=R_{j}+2 \mathbf{r}_{2}^{\prime} \cdot \boldsymbol{\epsilon}+\mathrm{O}\left(|\boldsymbol{\epsilon}|^{2}\right)$, i.e.

$$
\mathbf{r}_{2}^{\prime} \cdot \boldsymbol{\epsilon}=\mathrm{O}\left(|\boldsymbol{\epsilon}|^{2}\right) \text {. }
$$

Replacing $\mathbf{r}_{2}^{\prime}$ by $\mathbf{r}_{2}^{\prime}+\boldsymbol{\epsilon}$ in (2.11) yields (remember that $K_{i j k}=0$ for coaxial vectors)

$$
K_{i j k}=\left[\left(\mathbf{r}_{3}^{\prime}-\mathbf{r}_{1}^{\prime}\right) \times \boldsymbol{\epsilon}\right] \cdot V_{i j k}\left(\mathbf{r}_{1}^{\prime}, \mathbf{r}_{2}^{\prime}, \mathbf{r}_{3}^{\prime}\right)+\mathrm{O}\left(|\boldsymbol{\epsilon}|^{2}\right) .
$$

Clearly, if $\epsilon$ fulfills (2.12) and the r.h.s. of (2.13) does not vanish (which happens, for instance, if $\mathbf{r}_{2}^{\prime}$ and $\boldsymbol{\epsilon}$ are coplanar), so does $-\boldsymbol{\epsilon}$, so the kernel may have both signs, as stated.

A negative singularity is formally the worst defect a free energy density functional can have, because it implies that the absolute minimum of the free-energy functional is minus infinity, and it is reached for very singular density distributions. In practice, however, this defect can be overcome with a restricted minimization of the free energy within a functional family which does not include those singular distributions. Thus, the strong negative divergence of the original FMT proposal (1.11)-(1.14) for delta function density distributions, which precluded its use for crystals and narrow 0D cavities, does not interfere with its use (and very good results) for systems with planar symmetry, when the free energy is minimized with respect to planar density profiles $\rho_{i}(\mathbf{r})=\rho_{i}(z)$, independent of $x$ and $y$. The remaining negative divergences in our new version (2.13) are by far much weaker than those in (1.14), and they would be harmless for almost any virtual application of density functional theory with the variational minimization of the free energy constrained to families of functions (uniform in one or two variables, like in the adsorption at walls or in pores; periodic, like in freezing; etc.). This notwithstanding, we will show in the next section that the actual quantitative difference between this new functional and the former one (equations (1.16) and (1.17)) in applications to standard problems is negligible, the former functional being far simpler and easier to apply to polydisperse mixtures. 


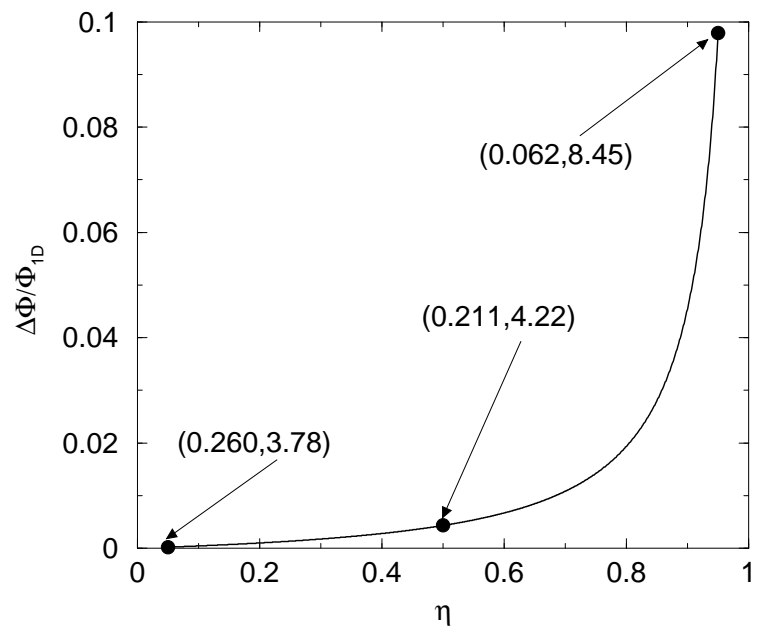

Figure 4. Maximum, with respect to the radii ratio $R_{l} / R_{s}$, and the relative packing fraction of the small segments, $x_{s} \equiv \eta_{s} / \eta$, of the relative error $\Delta \Phi / \Phi_{1 \mathrm{D}}$ of the one-dimensional reduction of functional (1.16) and (1.17) for a binary mixture, versus the total packing fraction, $\eta$, of the fluid. The values of the pair $\left(x_{s}, R_{l} / R_{s}\right)$ are given for three points of the curve. It can be seen that the maximum error occurs for $x_{s} \approx 0.2$ and $R_{l} / R_{s} \approx 4$ for most values of $\eta$.

\section{Results}

As stated in the previous section, the functional (1.16) and (1.17) does not recover the exact free energy of a uniform hard rod mixture when it is reduced to a one-dimensional density profile. The expression of the reduced functional is rather cumbersome, but in order to estimate this deviation we will simply evaluate the difference with respect to the exact value for a binary mixture. Let us denote respectively $\Phi$ and $\Phi_{1 \mathrm{D}}$ the reduced free energy density (in units of $k_{B} T$ ) and the exact free energy density, and define $\Delta \Phi=\Phi_{1 \mathrm{D}}-\Phi$. The relative error $\Delta \Phi / \Phi_{1 \mathrm{D}}$ has, for any packing fraction $\eta$, an absolute maximum as a function of the relative packing fraction of the small segments, $x_{s} \equiv \eta_{s} / \eta$ and the radii ratio, $R_{L} / R_{s}$. This maximum value is plotted vs. $\eta$ in figure 4 . In figure 5 we also plot the relative error $\Delta \Phi / \Phi_{1 \mathrm{D}}$ corresponding to $\eta=0.5$ both vs. $x_{s}$ at fixed $R_{L} / R_{s}$ (a) and vs. $R_{L} / R_{s}$ at fixed $x_{s}(\mathrm{~b})$, for the values corresponding to the maximum relative error. Notice that this error remains smaller than $2 \%$ up to $\eta=0.8$, and it is never larger than $10 \%$ even for packing fractions as high as $\eta=0.95$.

The conclusion we extract from these figures is that, despite having an incorrect dimensional crossover to $1 \mathrm{D}$, the functional (1.16), (1.17) produces results of high accuracy in this limit. A second test can be obtained by applying this functional and the one with the correction (2.10) to obtain the density profiles of a binary mixture of hard spheres both, near a hard wall and within a slit. The parameters of the system have been chosen in order to obtain the most prominent differences. The results are shown in figure 6, for the case of the hard wall, and figure 7 for the case of the slit. We see again that differences are negligible.

In view of these numerical tests we conclude that functional (1.16), (1.17) is preferable to the corrected one, despite its inability to recover the one-dimensional free energy exactly, simply because its structure is far simpler and more suitable to 


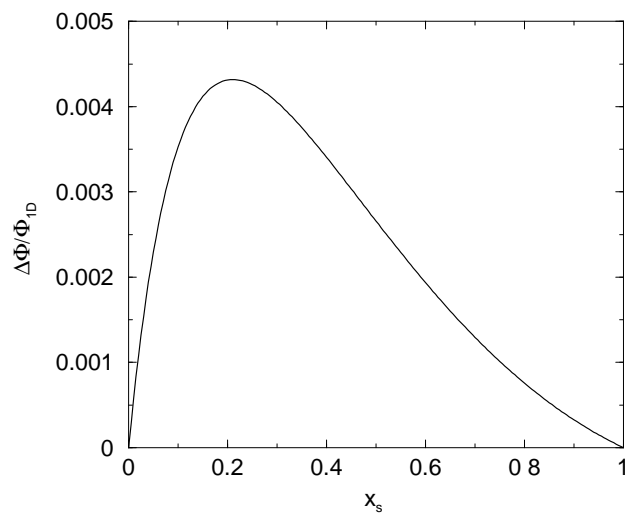

(a)

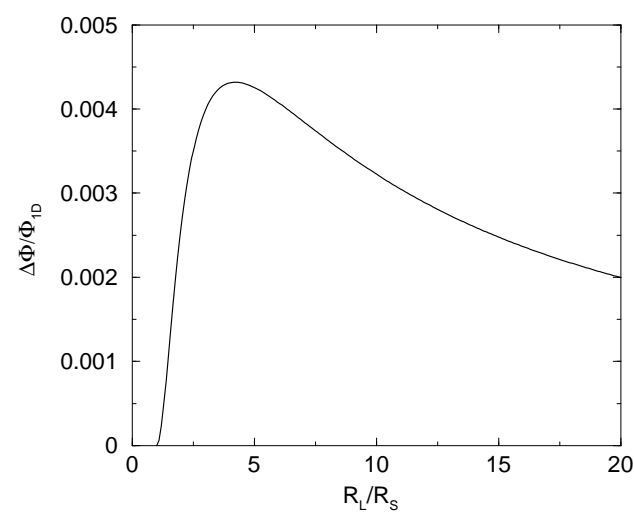

(b)

Figure 5. Relative error $\Delta \Phi / \Phi_{1 \mathrm{D}}$ of the one-dimensional reduction of functional (1.16) and (1.17) for a binary mixture at total packing fraction $\eta=0.5$, versus the relative packing fraction of the small segments, $x_{s} \equiv \eta_{s} / \eta$, for a radii ratio $R_{l} / R_{s}=4.22$ (a) as well as versus the radii ratio, $R_{l} / R_{s}$, for a value of $x_{s}=0.211$ (b).

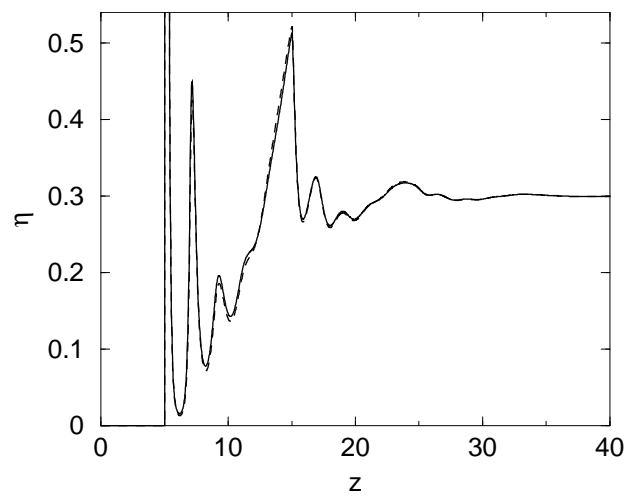

(a)

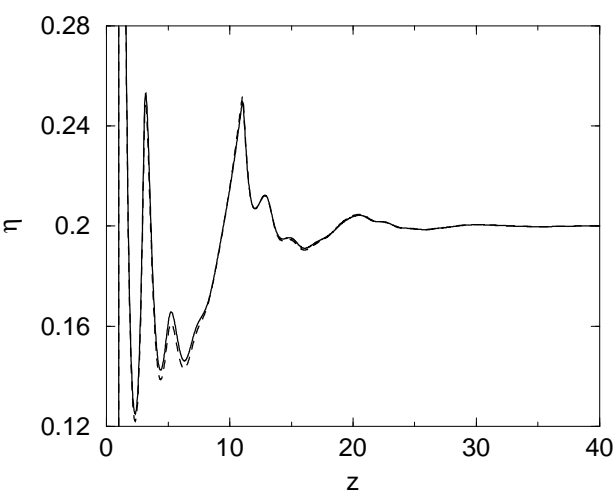

(b)

Figure 6. Density profiles of a binary mixture of hard spheres of radii $R_{-}=1$ and $R_{l}=5$ near a hard wall, as obtained both with functional (1.16), (1.17) only (dashed line) and with the correction (2.10) (solid line). The densities are multiplied by the volume of each type of particle. The bulk packing fractions of the small and large spheres are, respectively, $\eta_{s}=0.2$ and $\eta_{l}=0.3$. The difference between the results of both functionals can hardly be seen in the plotting scale.

be applied to polydisperse mixtures (see for instance 11]).

\section{Conclusions}

The main conclusion of the analysis we have performed on the construction of a fundamental measure functional for mixtures of hard spheres is that, as stated in the title, we appear to be 'close to the edge' of fundamental measure theory, in the sense that its internal structure seems almost exhausted. There are two main drawbacks 


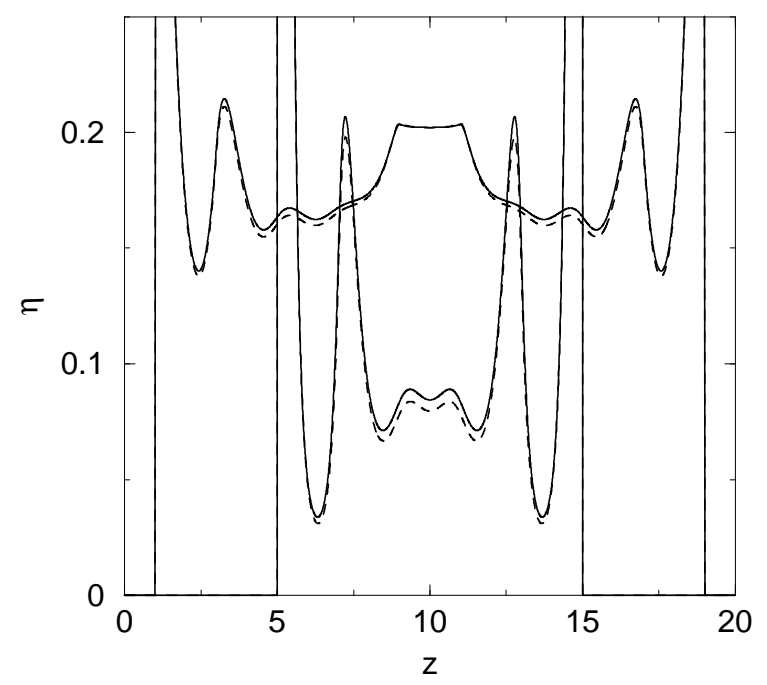

Figure 7. The same as in figure 6 but for a slit of length $20 R_{s}$. The system is in chemical equilibrium with a reservoir with packing fractions $\eta_{s}=0.2$ and $\eta_{l}=0.2$. Although the difference is now more visible, it is still negligible.

that seem unavoidable within the present scheme, both related to the structure of the third term $\Phi_{3}[\rho]$ in (1.11). The first problem is common to all the FMT versions and it concerns the existence of 'lost cases', i.e. the fact that configurations of three spheres with pairwise overlap but no triple overlap do not make any contribution to the functional. This reflects in that the lowest order of $c_{3}$ is incorrectly predicted (it vanishes for the lost cases, where it should still be 1 ). The obvious way to repair this defect is to introduce two point measures joining two 'halves' of Mayer functions. This would be a qualitative departure from the FMT structure, which would recover the exact $c_{3}$ at low density, but the increase in computational complexity would be considerable. Another way to circumvent this problem, while keeping the FMT structure and low computational cost, would be to replace the delta functions in the definition of $\Phi_{3}[\rho]$ by some other functions having a 'tail' extending beyond the radii of the particles (and presumably vanishing when particles no longer overlap). Although we cannot definitely exclude this possibility, we have tried several functional forms without success. This seems to be a too drastic change within the fundamental measure scheme, so much that adding such tails would 'break down' the functional at some other point. In particular, the dimensional crossover (to the exact 1D limit and to $0 \mathrm{D}$ cavities, strongly related to the density distribution in crystals) is a very stringent, hence fragile, requirement for an approximate functional, so virtually any modification can spoil it.

The existence of these lost cases forces to overweight the other configurations in order to compensate for the nonexistent ones and guarantee a reasonable equation of state. But as figure 1 and figure 2 show, this compensation introduces a dramatic bias in the case of mixtures of very dissimilar spheres. This may, at least in principle, have important consequences for the depletion effect in these systems and thus may affect phase behaviour, at least in its use to describe crystalline phases. Although in 
the application to planar density profiles, the effect would be strongly reduced by the averaging over the transverse directions.

The problem of the lost cases seems to be inherent to the fundamental measure structure with a notable exception: parallel hard parallelepipeds [4]. The peculiar shape of these bodies makes pairwise overlap and triple overlap to be equivalent conditions, so there are no lost cases for this particular form of the particles, and hence the lowest order in $c_{3}$ is exactly recovered.

The second problem, also associated to $\Phi_{3}[\rho]$, depends on the choice of the kernel function $K_{i j k}\left(\mathbf{r}_{1}^{\prime}, \mathbf{r}_{2}^{\prime}, \mathbf{r}_{3}^{\prime}\right)$ in (2.1), which marks the difference between the FMT versions proposed in the literature. The balance here comes between the computational cost and the existence of spurious divergences (of positive or negative sign) in the free energy. The original form proposed by Rosenfeld factorizes in terms of the scalar and vector weighted densities, but the negative divergences show up in the reduction to $1 \mathrm{D}$ and to $0 \mathrm{D}$ distributions. Simpler versions (for the monocomponent case) using no kernel function and only the scalar weighted density [3] give much stronger divergences (showing up even at the bulk fluid direct correlation function $c\left(r, \rho_{0}\right)$ ) but always with positive sign, so that in the minimization of the free energy those configurations contributing to the divergences are avoided, e.g. creating spurious kinks in the density profiles of a wall-fluid interface, but without the qualitative breakdown of (2.5) for strongly confined systems.

In contrast, the FMT version based on the exact reduction to the 0D limit 15] would have no divergences of the free energy at all, but it has a non-separable kernel with enormous computational cost (implying the multiple integrals over the densities at three different points). The proposal made in 15] and used by Groh and Mulder [17] of taking $K_{i j k} \sim\left[\mathbf{r}_{1}^{\prime} \cdot\left(\mathbf{r}_{2}^{\prime} \times \mathbf{r}_{3}^{\prime}\right)\right]^{2}$ would also kill all the divergences at the boundary between overlapping and non-overlapping spheres, and be separable in terms of the scalar, vector and a rank-two tensor weighted densities. However, this form gives a too smooth boundary behaviour for (2.1), since the kernel vanishes as the function $Z$ in the square root of denominator in (2.3), while the step-like dependence would require a non-separable kernel proportional to $K_{i j k} \sim\left|\mathbf{r}_{1}^{\prime} \cdot\left(\mathbf{r}_{2}^{\prime} \times \mathbf{r}_{3}^{\prime}\right)\right|$. The alternative kernel (2.6) and DF (1.16), proposed and successfully used [16] for monocomponent HS systems, achieved the separability (in term of the same set of weighted densities) and the sharpness (with the exact contribution to the triangle diagram for the bulk fluid), at the price of unleashing some weak divergences of positive sign of the free energy for some peculiar configurations of non-aligned molecules. The effect of those divergences would be extremely difficult to observe and the quality of that DF approximation seems to be limited mainly by the quality of the Percus-Yevick equation of state for the bulk HS fluid, and the associated condition of having a direct correlation function with the range of the hard core diameter 18], which is also intrinsic to the FMT.

The extension to HS mixtures imposes more severe restrictions, since the form (2.6) for HS of unequal size has (positive) divergences for peculiar aligned configurations which were not present for equal sized HS and which give stronger contributions than those of non-aligned configurations. Their main drawback is to spoil the exact reduction to the $1 \mathrm{D}$ limit, which was one of the most remarkable achievements for the monocomponent case. We have introduced here a new kernel form (2.7) which would recover that dimensional reduction for HS mixtures, at the price of introducing a new rank-three tensorial weighted density and creating some weak negative divergences for some non-aligned configurations. Besides, the radiidependent coefficients of this new kernel are not simply products of powers of the radii, 
but more complicated rational functions, so it turns out to be computationally more involved in applications to, for instance, polydisperse mixtures. Other possibilities remain for a candidate kernel, like squaring the one we propose, conveniently adjusting the coefficients; however this forces the introduction of very high rank tensor weights and removes the discontinuity of $c_{3}$, thus creating both computational and structural problems.

Altogether, the systematic improvement of the free energy density functional within the FMT seems to be frustrated by incompatible requirements, deeply rooted in the structure of the theory. Thus, one has to choose a functional form on the basis of its performance for the required practical use. The original proposal by Rosenfeld is quite accurate for systems with planar symmetry, for which the improvement obtained with the newer versions is purely marginal. The FMT version (1.16), with a moderate increase of the computational cost due to the presence of the tensorial weighted density (1.15), would give similar results as (1.14) for planar systems but it is also good for crystalline phases or other strongly confined density distributions. In the 1D limit it does not recover the exact DF for arbitrary distributions, but making a rather small error unless the 1D system is very close to the close packing limit. The extra computational cost of the FMT version (2.10), with a rank-three tensor and nonadditivity of the weighted densities in terms of a few moments of the radii, would only be worth for problems in which the exact $1 \mathrm{D}$ reduction is crucial and the spurious negative divergences of this free energy DF are made innocuous by the parametrization of the density distribution. It seems very unlikely that these two constrains appear in a practical problem, so that probably $(1.16)$ is the best practical choice.

To conclude, we have to point that the problems and limitations of the FMT density functionals, which we have explored here, should be considered in the right perspective, as we are asking the FMT functionals to perform well in problems which would be out of question for any other type of DF approximation. The exact (or nearly exact) reduction from three dimensional density distributions to the 1D limit, and the accurate behaviour in very narrow cavities, which opens the description of the crystalline phases with unconstrained density distributions, are fully out of range for the WDA or other DF approximations. The presence of spurious divergences in the FMT is the price to be paid for the sharpness of its non-local dependence, compared with the blurred dependence of the other DF approaches. The taming of those divergences to make them innocuous has been the 'holy grail' of the workers in the field, starting from Rosenfeld's choice for the combinations of $n_{i}(\mathbf{r})$ and $\mathbf{v}_{i}(\mathbf{r})$ in (1.13) and (1.14). The 'earthly' requirements of practical computability make that 'grail' unreachable, but the new versions explored here seem to be close enough to it for most practical purposes, providing by far the best density functional approximations for systems of HS mixtures.

\section{Acknowledgments}

This work is part of the research projects PB97-1223-C02-01 (DGESIC), BFM20011679-C03-02 (DGI) and BFM2000-0004 (DGI) of the Ministerio de Ciencia y Tecnología (Spain). Yuri Martínez-Ratón acknowledges financial support from a postdoctoral grant of the Dirección General de Investigación of the Consejería de Educación de la Comunidad de Madrid (Spain). 


\section{References}

[1] Rosenfeld Y 1989 Phys. Rev. Lett. 63980

1993 J. Chem. Phys. 988126

-1994 Phys. Rev. E 50 R3318

1996 J. Phys.: Condens. Matter 89287

1996 J. Phys.: Condens. Matter 8 L795

[2] Kierlik E and Rosinberg M L 1990 Phys. Rev. A 423382

-1991 Phys. Rev. A 445025

Phan S, Kierlik E, Rosinberg M L, Bildstein B and Kahl G 1993 Phys. Rev. E 48618

[3] González A, White J A and Evans R 1997 J. Phys.: Condens. Matter 92375

[4] Cuesta J A 1996 Phys. Rev. Lett. 763742

Cuesta J A and Martínez-Ratón Y 1997 Phys. Rev. Lett. 783681 -1997 J. Chem. Phys. 1076379

Martínez-Ratón Y and Cuesta J A 1998 Phys. Rev. E 58 R4080

1999 J. Chem. Phys. 1111317

[5] Schmidt M 1999 Phys. Rev. E 60 R6291

-2000 Phys. Rev. Lett. 851934

2000 Phys. Rev. E 63 010101(R)

2001 Phys. Rev. E 63 050201(R)

[6] Hansen J P and McDonald I R 1986 Theory of Simple Liquids 2nd ed. (London: Academic Press)

[7] Tarazona P 1985 Phys. Rev. A 31, 2672

[8] Curtin W A and Ashcroft N W 1989 Phys. Rev. A 322909

[9] Percus J K and Yevick G J 1958 Phys. Rev. 1101

Thiele E 1963 J. Chem. Phys. 39474

Wertheim M S 1963 Phys. Rev. Lett. 10321

[10] Percus J K 1976 J. Stat. Phys. 15505

[11] Pagonabarraga I, Cates M E and Ackland G J 2000 Phys. Rev. Lett. 84911

[12] Rosenfeld Y 1990 Phys. Rev. A 42, 5978

[13] Lutsko J F and Baus M 1990 Phys. Rev. A 41, 6647

Tejero C F, Ripoll M S and Pérez A 1995 Phys. Rev. E 523632

[14] Rosenfeld Y, Schmidt M, Löwen H and Tarazona P 1997 Phys. Rev. E 554245

[15] Tarazona P and Rosenfeld Y 1997 Phys. Rev. E 55 R4873

1999 New Approaches to Problems in Liquid State Theory ed C Caccamo et al. (Dordrecht: Kluwer) p 293

[16] Tarazona P 2000 Phys. Rev. Lett. 84694

[17] Groh B and Mulder B 2000 Phys. Rev. E 613811

Groh B 2000 Phys. Rev. E 615218

[18] Tarazona P 2002 Physica A 306243 